\title{
Validation of the Korean Liver Cancer Association-National Cancer Center 2018 Criteria for the Noninvasive Diagnosis of Hepatocellular Carcinoma Using Magnetic Resonance Imaging
}

\author{
Sunyoung Lee, Myeong-Jin Kim \\ Department of Radiology and Research Institute of Radiological Science, Severance Hospital, Yonsei University College of Medicine, \\ Seoul, Korea
}

Received Jan. 20, 2020

Revised Mar. 16, 2020

Accepted Mar. 18, 2020
Background/Aims: This study aimed to assess the validity and diagnostic performance of the imaging criteria of Korean Liver Cancer Association-National Cancer Center (KLCA-NCC) 2018 using magnetic resonance imaging (MRI) in high-risk patients for HCC.

Methods: This retrospective study included 142 treatment-naïve patients (81 patients who underwent MRI with extracellular contrast agent and 61 who underwent MRI with hepatobiliary agent; 183 lesions including $149 \mathrm{HCCs}$ ) with a high risk of HCC who underwent multiphasic contrast-enhanced MRI from January to December 2015. All lesions were categorized according to the KLCA-NCC 2018 imaging diagnostic criteria by two readers, and per-lesion diagnostic performances were compared.

Results: According to the KLCA-NCC 2018, none (0\%) of the 13 benign category lesions, 11 (44.0\%) of 25 indeterminate category lesions, 15 (93.8\%) of 16 probable HCC category lesions, and $97(99.0 \%)$ of 98 definite HCC category lesions were ultimately diagnosed as HCCs. The sensitivity and specificity of definite HCC category were $65.1 \%$ and $97.1 \%$, respectively, and those of the combination of definite and probable HCC categories were $75.2 \%$ and $94.1 \%$, respectively. The sensitivity of the combination of definite and probable HCC categories was significantly higher than that of definite HCC $(P<0.001)$, but the specificity was not significantly lower $(P>0.999)$.

Conclusions: The noninvasive imaging diagnosis of KLCA-NCC 2018 on MRI is reliable and useful for diagnosing $\mathrm{HCC}$ in high-risk patients. Combining definite and probable $\mathrm{HCC}$ categories of KLCA-NCC 2018 improves the sensitivity while maintaining a high specificity. (J Liver Cancer 2020;20:120-127)

Keywords: Hepatocellular carcinoma; Diagnosis; Sensitivity and specificity; Magnetic resonance imaging

\section{Corresponding author : Sunyoung Lee}

Department of Radiology and Research Institute of Radiological Science, Severance Hospital, Yonsei University College of Medicine, 50-1 Yonseiro, Seodaemun-gu, Seoul 03722, Korea

Tel. +82-2-2228-7400; Fax. +82-2-2227-8337

E-mail; carnival0126@gmail.com

https://orcid.org/0000-0002-6893-3136

\section{INTRODUCTION}

Hepatocellular carcinoma (HCC) is the most common primary hepatic malignancy. ${ }^{1}$ HCC is the only malignancy that can be diagnosed based on imaging without the need for pathologic confirmation in high-risk patients. Thus, reliable noninvasive imaging criteria are essential. ${ }^{2-4}$ 
The Korean Liver Cancer Study Group (KLCSG)-National Cancer Center (NCC) Korea practice guidelines for HCC were first developed in 2003, and subsequently revised in 2009, 2014, and 2018. . $^{5}$ They provide diagnosis and treatment guidelines specific to the Asian population, particularly Korean. ${ }^{5,6}$ In contrast to the previous version of the KLCSGNCC Korea practice guidelines, the recently updated Korean Liver Cancer Association (KLCA)-NCC 2018 practice guideline for the imaging diagnosis of HCC has been revised into a non-binary decision algorithm that addresses the full spectrum of liver lesions, ranging from benign to malignant. ${ }^{7}$ KLCA-NCC 2018 categorizes a lesion as benign, indeterminate, probable HCC, definite HCC, or targetoid appearance based on major, ancillary, and targetoid imaging features. ${ }^{6,8}$

However, to the best of our knowledge, external validation of the imaging criteria of KLCA-NCC 2018 has not been published. Therefore, the aim of this study was to assess the validity and diagnostic performance of the imaging criteria of KLCA-NCC 2018 using magnetic resonance imaging (MRI) in high-risk patients for HCC.

\section{METHODS}

This retrospective study was approved by the institutional review board of Severance Hospital (IRB number 4-20191218), and the requirement for informed consent was waived.

\section{Study population}

We retrospectively searched our institution's databases for a clinical cohort of HCC surveillance and identified 1,211 patients who underwent MRI for diagnostic workup between January 2015 and December 2015. The inclusion criteria were treatment-naïve adult patients ( $\geq 18$ years) who were at a high risk for HCC according to the KLCA-NCC 2018 with at least one and up to five hepatic observations (each $\geq 1$ $\mathrm{cm}$ ) on MRI. We excluded patients with insufficient final diagnosis such as unknown final diagnosis of malignancy as a result of immediate locoregional therapy or insufficient follow-up ( $<2$ years) for benign lesions to determine size stabil- ity. We did not include observations such as hepatic cysts, perfusion alteration, hepatic fat deposition or sparing, hypertrophic pseudomass, confluent fibrosis, and focal scar. A total of 142 patients (mean age, 57.2 years; 104 men and 38 women) with 183 lesions (149 HCCs, 6 non-HCC malignancies, and 28 benign lesions) met our criteria and were included in this study.

\section{MRI examination}

MRI was performed using one of the three 3.0-T systems (Magnetom Trio Tim, Siemens Medical Solutions, Erlangen, Germany; Intera Achieva, Ingenia, or Ingenia CX, Philips Medical Systems, Best, The Netherlands; and Discovery MR 750w, GE Medical Systems, Waukesha, WI, USA) or a 1.5-T system (Intera Achieva, Philips Medical Systems, Best, The Netherlands). The protocol included the acquisition of dualecho T1-weighted gradient-echo images (in-phase and opposed-phase), T1-weighted three-dimensional gradient-echo images with dynamic contrast-enhancement, navigator-triggered single or multi-shot T2-weighted images, and diffusionweighted images at $b$-values of 0 or 50,400 , and $800 \mathrm{~s} / \mathrm{mm}^{2}$. Dynamic T1-weighted imaging was performed before and after administering one of the two extracellular contrast agents (ECAs) (gadoterate meglumine, Dotarem, Guerbet SA, Aulnay-sous-Bois, France; and gadopentetate dimeglumine, Magnevist, Bayer Pharma AG, Berlin, Germany) or a hepatobiliary agent (HBA) (gadoxetate disodium, Primovist, Bayer Pharma AG): a bolus injection of $0.2 \mathrm{~mL} / \mathrm{kg}$ gadoterate meglumine or gadopentetate dimeglumine $(n=81)$ at a rate of 2.0 $\mathrm{mL} / \mathrm{s}$, or $0.1 \mathrm{~mL} / \mathrm{kg}$ gadoxetate disodium $(\mathrm{n}=61)$ at a rate of $1.0 \mathrm{~mL} / \mathrm{s}$, followed by a $20-\mathrm{mL}$ saline flush using a power injector. Arterial phase scanning was initiated using the test-bolus or bolus-tracking technique, and the portal venous phase (PVP) (60 s), 3-min delayed phase (DP; transitional phase on HBA-MRI), and 20-min hepatobiliary phase (HBP) images (only after HBA administration) were obtained.

\section{Image analysis}

Two board-certified abdominal radiologists independently 
reviewed all images. They were blinded to the final diagnoses of all lesions but knew that the study population comprised patients at high risk for HCC. According to the KLCA-NCC 2018, the readers assessed the presence or absence of major and ancillary imaging features and targetoid appearance. ${ }^{6,8}$ Each lesion was categorized as definite HCC, probable HCC, indeterminate, benign, or targetoid appearance. ${ }^{6,8}$ Definite HCC was defined as arterial phase hyperenhancement with washout in the PVP, DP, or HBP. ${ }^{6,8}$ In a lesion with some but not all of the major imaging features of HCC, probable HCC was assigned only when the lesion fulfilled at least one item from each of the following two categories of ancillary imaging features: favoring malignancy in general, and favoring HCC in particular. ${ }^{6,8}$ When the imaging diagnosis was inconclusive, a lesion was defined as indeterminate. ${ }^{6,8} \mathrm{~A}$ lesion that fulfilled at least one of the ancillary features favoring benignity was classified as a benign lesion. ${ }^{6,8}$ Targetoid appearance was evaluated on diffusion-weighted imaging (DWI) or contrast-enhanced sequences. After an independent categorization, inter-reader agreement was evaluated. In cases of discrepancies between the two readers, the final category was determined by consensus discussion.

\section{Reference standards}

The diagnoses of HCCs and non-HCC malignancies were confirmed by pathology, including surgical resection $(n=142)$ or explant for transplantation $(n=13)$. Benign diagnoses were obtained through pathology $(n=1)$ or typical imaging features and stability at imaging for at least 2 years $(\mathrm{n}=27)$. The mean interval between MRI and pathological confirmation was 19.2 (range, 1-58) days.

\section{Statistical analysis}

All analyses were performed on a per-lesion basis. Per-lesion estimates of diagnostic performances (sensitivity, specificity, positive predictive value [PPV], and negative predictive value $[\mathrm{NPV}])$ were calculated. The sensitivities and specificities of definite HCC category and the combination of definite and probable HCC categories of KLCA-NCC 2018 were compared using the McNemar's test. The $\chi^{2}$ test was used to compare the diagnostic performances of definite HCC and the combination of definite and probable HCC of KLCA-NCC 2018 between the two independent ECA-MRI and HBA-MRI groups. Inter-reader agreement was evaluated using Cohen $\kappa$ coefficient. The $\kappa$-value (the level of agreement) was defined as follows: poor, 0.00-0.20; fair, 0.21-0.40; moderate, 0.41-0.60; good, 0.61-0.80; and excellent, 0.811.00. Statistical analyses were performed using MedCalc version 16.2.1 (MedCalc Software, Ostend, Belgium).

Table 1. Characteristics of the patients and lesions

\begin{tabular}{|c|c|}
\hline Characteristic & Value \\
\hline Patients & $n=142$ \\
\hline Sex (male) & $104(73.2)$ \\
\hline Age (years) & $57.2 \pm 9.9(29-78)$ \\
\hline \multicolumn{2}{|l|}{ Etiology of liver disease } \\
\hline Hepatitis B & $119(83.8)$ \\
\hline Hepatitis C & $8(5.6)$ \\
\hline Alcohol & $12(8.5)$ \\
\hline Others & $3(2.1)$ \\
\hline Lesions & $n=183$ \\
\hline Lesion size (mm) & $30.2 \pm 23.9(10-200)$ \\
\hline \multicolumn{2}{|l|}{ MRI contrast } \\
\hline ECA-MRI & $104(56.8)$ \\
\hline HBA-MRI & $79(43.2)$ \\
\hline \multicolumn{2}{|l|}{ Final diagnosis } \\
\hline $\mathrm{HCC}$ & $149(81.4)$ \\
\hline \multicolumn{2}{|l|}{ Non-HCC malignancy } \\
\hline $\begin{array}{l}\text { Combined hepatocellular- } \\
\text { cholangiocarcinoma }\end{array}$ & $4(2.2)$ \\
\hline Intrahepatic cholangiocarcinoma & $2(1.1)$ \\
\hline \multicolumn{2}{|l|}{ Benign lesion } \\
\hline Hemangioma & $6(3.3)$ \\
\hline Focal nodular hyperplasia-like nodule & $7(3.8)$ \\
\hline Regenerative nodule & $5(2.7)$ \\
\hline Dysplastic nodule & $10(5.5)$ \\
\hline
\end{tabular}

Values are presented as mean \pm standard deviation (range) or number (\%) unless otherwise indicated.

MRI, magnetic resonance imaging; ECA, extracellular contrast agent; HBA, hepatobiliary agent; HCC, hepatocellular carcinoma. 


\section{RESULTS}

\section{Patients and lesions}

The characteristics of the patients and lesions are summarized in Table 1. Our study included 81 patients with 104 lesions who underwent ECA-MRI, and 61 patients with 79 lesions who underwent HBA-MRI. Hepatitis B (83.8\%) was the most common etiology of liver disease. The 183 lesions comprised 149 (81.4\%) HCCs, 6 (3.3\%) non-HCC malignancies, and 28 (15.3\%) benign lesions.

\section{Lesion categorization according to the KLCA- NCC 2018}

Lesion categorization according to the KLCA-NCC 2018 is summarized in Table 2. Thirteen (7.1\%) lesions were classified as benign, $25(13.7 \%)$ as indeterminate, $16(8.7 \%)$ as probable HCC, and 98 (53.6\%) as definite HCC. Thirty-one (16.9\%) lesions were categorized as targetoid appearance.
All lesions classified as benign category on MRI were finally diagnosed as benign lesions. Eleven (44.0\%) of 25 lesions classified as indeterminate category were ultimately diagnosed as HCCs. Fifteen (93.8\%) of 16 lesions classified as probable HCC were finally diagnosed as HCCs. Ninety-seven (99.0\%) of 98 lesions classified as definite HCC were ultimately diagnosed as HCCs.

\section{Diagnostic performances of KLCA-NCC 2018 on MRI for HCC}

The diagnostic performances of KLCA-NCC 2018 on MRI for HCC are summarized in Table 3. Considering only lesions classified as definite HCC, the sensitivity, specificity, PPV, and NPV were 65.1\%, 97.1\%, 99.0\%, and 38.8\%, respectively (Fig. 1, 2). There was one false-positive diagnosis of KLCA-NCC 2018 in the definite HCC category, which was a combined hepatocellular-cholangiocarcinoma. Considering definite and probable HCC categories, the sensitivity, specificity, PPV, and NPV were 75.2\%, 94.1\%, 98.3\%,

Table 2. Lesion categorization according to the KLCA-NCC 2018

\begin{tabular}{lcccc}
\hline \multirow{2}{*}{ Category } & $\begin{array}{c}\text { Total No. } \\
\text { of lesions }\end{array}$ & HCC & Non-HCC malignancy & Benign lesions \\
\cline { 3 - 5 } & 13 & 0 & 0 & 13 \\
\hline Benign & 25 & 11 & 0 & 14 \\
Indeterminate & 16 & 15 & 0 & 1 \\
Probable HCC & 98 & 97 & 1 & 0 \\
Definite HCC & 31 & 26 & 5 & 0 \\
Targetoid appearance & & & & 1 \\
\hline
\end{tabular}

KLCA-NCC, Korean Liver Cancer Association-National Cancer Center; HCC, hepatocellular carcinoma.

Table 3. Diagnostic performances of definite HCC and definite and probable HCC categories according to the KLCA-NCC 2018

\begin{tabular}{lccc}
\hline & $\begin{array}{c}\text { Definite HCC of KLCA-NCC } \mathbf{2 0 1 8} \\
(\mathbf{9 5 \%} \mathbf{~ C l})\end{array}$ & $\begin{array}{c}\text { Definite+probable HCC of KLCA- } \\
\text { NCC 2018 (95\% Cl) }\end{array}$ & P-value $^{*}$ \\
\hline Sensitivity & $65.1(56.9-72.7)$ & $75.2(67.4-81.9)$ & $<0.001$ \\
Specificity & $97.1(84.7-99.9)$ & $94.1(80.3-99.3)$ & $>0.999$ \\
PPV & $99.0(94.5-99.9)$ & $98.3(93.8-99.8)$ & - \\
NPV & $38.8(28.4-50.0)$ & $46.4(34.3-58.8)$ & - \\
\hline
\end{tabular}

HCC, hepatocellular carcinoma; KLCA-NCC, Korean Liver Cancer Association-National Cancer Center; Cl, confidence interval; PPV, positive predictive value; NPV, negative predictive value.

" $P$-values between definite HCC category and the combination of definite and probable HCC categories of KLCA-NCC 2018 by using the McNemar's test. 

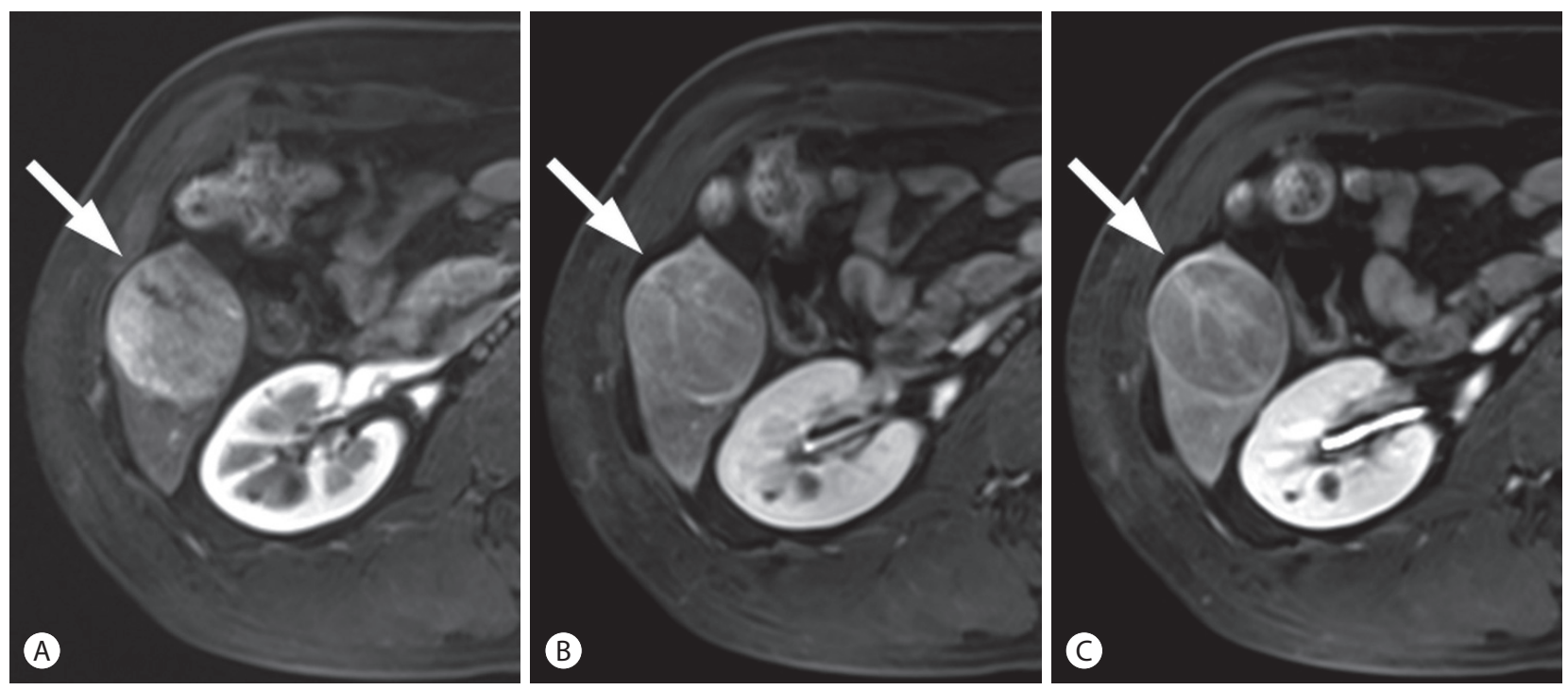

Figure 1. Hepatocellular carcinoma (HCC) in a 50-year-old man with chronic hepatitis B. In the arterial (A), portal venous (B), and delayed phase (C) images after the administration of extracellular contrast agent, a 47-mm liver mass (arrows) shows arterial phase hyperenhancement with washout in the delayed phase. The mass was categorized as definite HCC according to the Korean Liver Cancer Association-National Cancer Center 2018 criteria.
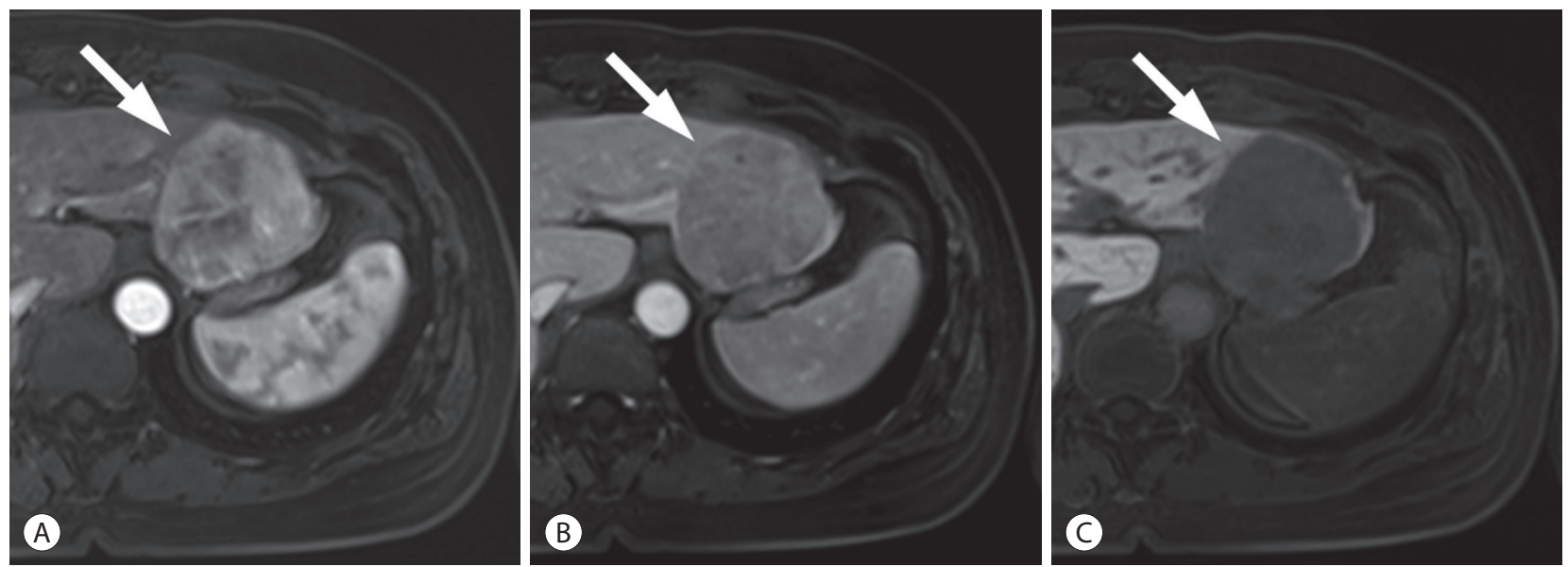

Figure 2. Hepatocellular carcinoma (HCC) in a 42-year-old woman with chronic hepatitis B. In the arterial (A), portal venous (B), and hepatobiliary phase (C) images after the administration of hepatobiliary agent, a 54-mm liver mass (arrows) shows arterial phase hyperenhancement with washout in the portal venous and hepatobiliary phases. The mass was categorized as definite HCC according to the Korean Liver Cancer Association-National Cancer Center 2018 criteria.

and $46.4 \%$ in diagnosing HCC, respectively (Fig. 3). The sensitivity of the combination of definite HCC and probable HCC was significantly higher than that of definite HCC $(P<0.001)$, but the specificity was not significantly lower $(P>0.999)$.
4. Comparison of diagnostic performances of ECA-MRI and HBA-MRI for definite HCC and definite and probable HCC categories according to the KLCA-NCC 2018

Table 4 presents the comparison of diagnostic performances of ECA-MRI and HBA-MRI for definite HCC and definite and probable HCC categories according to the 

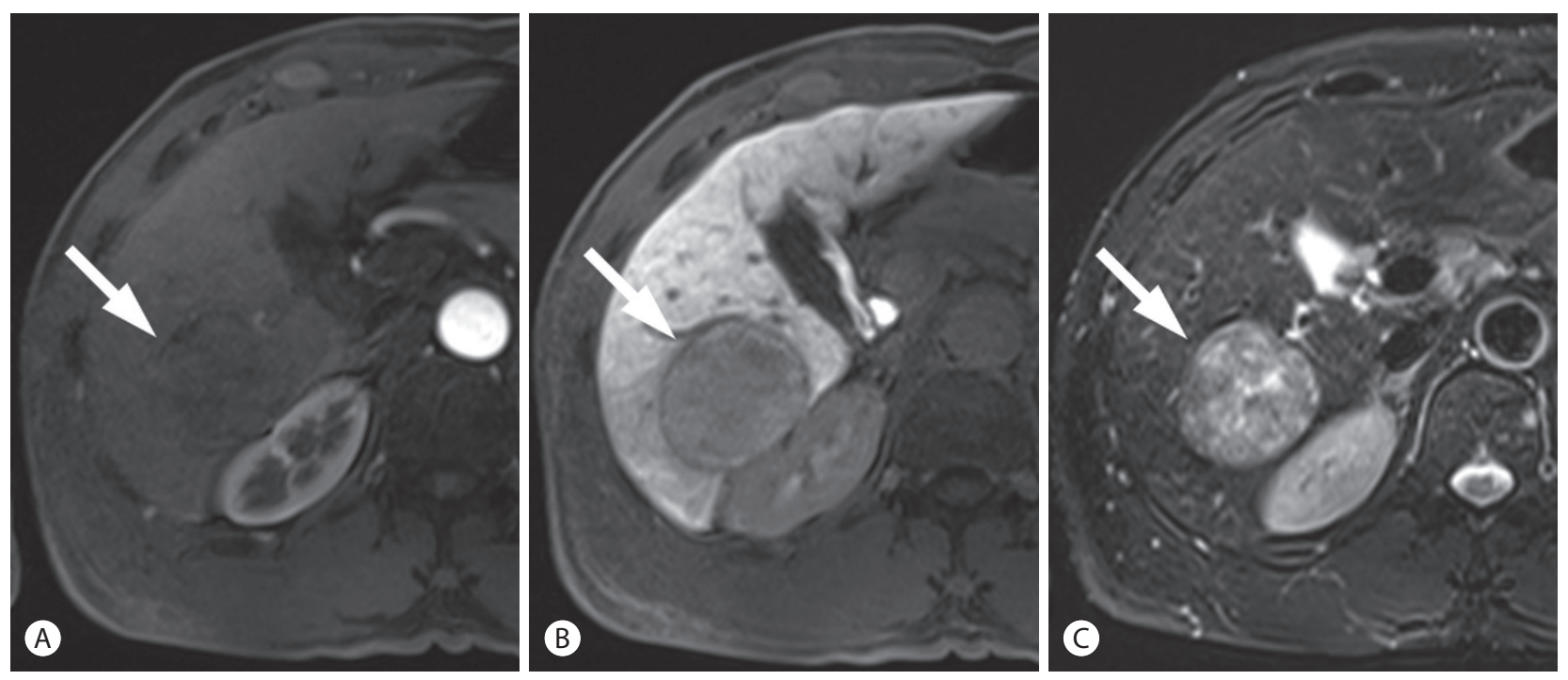

Figure 3. Hepatocellular carcinoma (HCC) in a 57-year-old man with chronic hepatitis B. In the arterial (A) and hepatobiliary phase (B) images after the administration of hepatobiliary agent, a $50-\mathrm{mm}$ liver mass (arrows) shows washout in the hepatobiliary phase without arterial phase hyperenhancement. Non-enhancing capsule is seen. On T2-weighted image (C), the mass demonstrates mild-to-moderate T2 hyperintensity. The mass was categorized as probable HCC according to the Korean Liver Cancer Association-National Cancer Center 2018 criteria.

Table 4. Comparison of diagnostic performances of ECA-MRI and HBA-MRI for definite HCC and definite and probable HCC categories according to the KLCA-NCC 2018

\begin{tabular}{|c|c|c|c|}
\hline & ECA-MRI $(95 \% \mathrm{Cl})$ & HBA-MRI (95\% CI) & $P$-value ${ }^{*}$ \\
\hline \multicolumn{4}{|c|}{ Definite HCC of KLCA-NCC 2018} \\
\hline Sensitivity & $60.5(49.0-71.2)$ & $70.6(58.3-81.0)$ & 0.198 \\
\hline Specificity & $95.7(78.1-99.9)$ & $100(71.5-100)$ & 0.483 \\
\hline PPV & $98.0(89.4-99.9)$ & $100(92.6-100)$ & 0.325 \\
\hline NPV & $40.7(27.6-55.0)$ & $35.5(19.2-54.6)$ & 0.632 \\
\hline \multicolumn{4}{|c|}{ Definite+probable HCC of KLCA-NCC 2018} \\
\hline Sensitivity & $74.1(63.1-83.2)$ & $76.5(64.6-85.9)$ & 0.736 \\
\hline Specificity & $95.7(78.1-99.9)$ & $90.9(58.7-99.8)$ & 0.582 \\
\hline PPV & $98.4(91.2-99.9)$ & $98.1(89.9-99.9)$ & 0.920 \\
\hline NPV & $51.2(35.5-66.7)$ & $38.5(20.2-59.4)$ & 0.305 \\
\hline
\end{tabular}

ECA, extracellular contrast agent; MRI, magnetic resonance imaging; HBA, hepatobiliary agent; HCC, hepatocellular carcinoma; KLCA-NCC, Korean Liver Cancer Association-National Cancer Center; Cl, confidence interval; PPV, positive predictive value; NPV, negative predictive value.

" $P$-values between ECA-MRI and HBA-MRI by using the $x^{2}$ test.

KLCA-NCC 2018. In definite HCC category of KLCA-NCC 2018, the sensitivity, specificity, PPV, and NPV were $60.5 \%$, $95.7 \%, 98.0 \%$, and $40.7 \%$ for ECA-MRI, and 70.6\%, 100\%, $100 \%$, and $35.5 \%$ for HBA-MRI, respectively. No statistically significant differences in the sensitivity, specificity, PPV, and NPV were found for definite HCC category between ECAMRI and HBA-MRI $(P>0.05$ for all).
When combining definite HCC and probable HCC categories, the diagnostic performances of ECA-MRI and HBAMRI did not significantly differ, with a sensitivity, specificity, PPV, and NPV of $74.1 \%, 95.7 \%, 98.4 \%$, and $51.2 \%$ for ECA-MRI, respectively, and 76.5\%, 90.9\%, 98.1\%, and $38.5 \%$ for HBA-MRI, respectively $(P>0.05$ for all). 


\section{Inter-reader agreement}

The inter-reader agreement for categorization of the lesions according to the KLCA-NCC 2018 was excellent $(\kappa=0.91,95 \%$ confidence interval, $0.87-0.95)$.

\section{DISCUSSION}

Our results demonstrated that noninvasive imaging diagnosis according to the KLCA-NCC 2018 on MRI is reliable and useful for diagnosing HCC in high-risk patients, providing a stratified probability of HCC and showing a high specificity and PPV for probable HCC and definite HCC categories. When combining definite HCC and probable HCC categories for the diagnosis of HCC, sensitivity was substantially increased, while maintaining the specificity.

In our study, no HCC diagnosis was reported for the benign category of KLCA-NCC 2018 imaging criteria, which reflects well-defined imaging features for benignity. For the indeterminate category, the proportion of HCC was $44.0 \%$. Because of the substantial probability of HCC, lesions in the indeterminate category should be closely followed up or be considered for biopsy. The proportion of HCC was 93.8\% for the probable HCC category and 99\% for the definite HCC category. These results demonstrate that the imaging criteria of KLCA-NCC 2018 could provide a stratified probability for HCC.

The present study confirmed the high specificity for HCC diagnosis using the KLCA-NCC 2018 imaging criteria. The diagnostic criteria for HCC according to the KLCA-NCC 2018, may have a high specificity by excluding hemangiomas or other malignancies, based on ancillary features favoring benignity such as marked T2 hyperintensity or targetoid appearance on DWI or contrast-enhanced sequences prior to the application of major imaging features. ${ }^{6,8}$ While the definite HCC category of KLCA-NCC 2018 shows a suboptimal sensitivity, the combination of definite and probable HCC categories provides improved sensitivity while maintaining a high specificity. Based on the KLCA-NCC 2018 criteria, a lesion classified as probable HCC requires at least one item from each of the two categories of ancillary imaging features (favoring malignancy in general and favoring HCC in partic- ular); ${ }^{6,8}$ thus, it may prevent significant loss of specificity.

In our study, there were no significant differences in the diagnostic performances of KLCA-NCC 2018 between ECAMRI and HBA-MRI. The updated versions of the imaging criteria for diagnosing HCC state that there is currently insufficient evidence to recommend one contrast agent type over the other. ${ }^{2,3}$

This study has several limitations. First, due to the retrospective nature of this study, there may have been a potential selection bias. Second, this study enrolled patients from a single center in a chronic hepatitis B endemic area and thus, the generalizability of the results may be limited. Third, the composite clinical reference standard for the determination of benign lesions was used. However, pathologic confirmation for highly suspected benign lesions is not recommended in clinical practice, and the application of a strict standard of reference (only pathology) for benign lesions may have resulted in a confirmation bias. Finally, our use of consensus categorization may have increased our accuracy statistics compared with the clinical single-reader scenario.

In conclusion, the noninvasive imaging diagnosis of KLCA-NCC 2018 on MRI is reliable and useful for diagnosing HCC in high-risk patients. Combining definite and probable HCC categories of KLCA-NCC 2018 improves the sensitivity while maintaining a high specificity.

\section{ACKNOWLEDGMENTS}

This study was supported by the Korean Liver Cancer Association Research Award (recipient S.L.).

\section{AUTHOR CONTRIBUTIONS}

-Study concept and design: S.L.

-Data acquisition: S.L. and M-J.K.

-Data analysis and interpretation: S.L.

-Drafting of the manuscript; critical revision of the manuscript for important intellectual content: S.L. and M-J.K.

-Statistical analysis: S.L.

-Administrative, technical, or material support; study supervision: S.L. 


\section{Conflicts of Interest}

The authors have no conflicts to disclose.

\section{REFERENCES}

1. El-Serag HB. Hepatocellular carcinoma. N Engl J Med 2011;365:11181127.

2. Marrero JA, Kulik LM, Sirlin CB, Zhu AX, Finn RS, Abecassis MM, et al. Diagnosis, staging, and management of hepatocellular carcinoma: 2018 Practice Guidance by the American Association for the Study of Liver Diseases. Hepatology 2018;68:723-750.

3. European Association for the Study of the Liver. European Association for the Study of the EASL Clinical Practice Guidelines: management of hepatocellular carcinoma. J Hepatol 2018;69:182-236.

4. Lee S, Kim MJ, Kim SS, Shin H, Kim DY, Choi JY, et al. Retrospective comparison of EASL 2018 and LI-RADS 2018 for the noninvasive diagnosis of hepatocellular carcinoma using magnetic resonance imaging. Hepatol Int 2020;14:70-79.

5. Korean Liver Cancer Study Group, National Cancer Center Korea. 2014 KLCSG-NCC Korea practice guideline for the management of hepatocellular carcinoma. Gut Liver 2015;9:267-317.

6. Korean Liver Cancer Association; National Cancer Center. 2018 Korean Liver Cancer Association-National Cancer Center Korea Practice Guidelines for the Management of Hepatocellular Carcinoma. Gut Liver 2019;13:227-299.

7. Kim TH, Kim SY, Tang A, Lee JM. Comparison of international guidelines for noninvasive diagnosis of hepatocellular carcinoma: 2018 update. Clin Mol Hepatol 2019;25:245-263.

8. Korean Liver Cancer Association; National Cancer Center. 2018 Korean Liver Cancer Association-National Cancer Center Korea Practice Guidelines for the Management of Hepatocellular Carcinoma. Korean J Radiol 2019;20:1042-1113. 